\title{
Supplementary material for A hierarchical Bayesian setting for an inverse problem in linear parabolic PDEs with noisy boundary conditions
}

F. Ruggeri, Z. Sawlan, M. Scavino and R. Tempone zaid.sawlan@kaust.edu.sa

In this supplementary material, we provide proofs of Theorem 7, Theorem 8 and Remark 14.

\section{Appendix A}

Proof of Theorem 7:

First, let us introduce the vectors

$$
\begin{aligned}
\underset{(I-1) \times 1}{F_{L, 1}} & =\left[-\left(\int_{x_{L}}^{x_{R}} \frac{x_{R}-x}{x_{R}-x_{L}} \phi_{j} d x\right)_{j}\right], \\
\underset{(I-1) \times 1}{F_{L, 2}} & =\left[\left(\int_{x_{L}}^{x_{R}}\left(\frac{x_{R}-x}{x_{R}-x_{L}}-\Delta t L_{\boldsymbol{\theta}} \frac{x_{R}-x}{x_{R}-x_{L}}\right) \phi_{j} d x\right)_{j}\right], \\
\underset{(I-1) \times 1}{F_{R, 1}} & =\left[-\left(\int_{x_{L}}^{x_{R}} \frac{x-x_{L}}{x_{R}-x_{L}} \phi_{j} d x\right)_{j}\right], \\
\underset{(I-1) \times 1}{F_{R, 2}} & =\left[\left(\int_{x_{L}}^{x_{R}}\left(\frac{x-x_{L}}{x_{R}-x_{L}}-\Delta t L_{\boldsymbol{\theta}} \frac{x-x_{L}}{x_{R}-x_{L}}\right) \phi_{j} d x\right)_{j}\right],
\end{aligned}
$$


and the matrices

$$
\begin{aligned}
& \underset{(I-1) \times(I-1)}{\mathbf{B}}=\left(M+\Delta t S_{\boldsymbol{\theta}}\right)^{-1} M \\
& \underset{(I-1) \times N+1}{\mathbf{F}_{L, n}}=\left(\begin{array}{cccc}
\underset{(I-1) \times(n-1)}{\mathbf{0}} & F_{L, 1} & F_{L, 2} & \underset{(I-1) \times 1}{\mathbf{0}} \\
(I-1) \times 1 & (I-1) \times(N-n)
\end{array}\right), n=2, \ldots, N-1, \\
& \underset{(I-1) \times N+1}{\mathbf{F}_{R, n}}=\left(\begin{array}{cccc}
\underset{(I-1) \times(n-1)}{\mathbf{0}} & F_{R, 1} & F_{R, 2} & \underset{(I-1) \times 1}{\mathbf{0}} \\
(I-1) \times 1 & (I-1) \times(N-n)
\end{array}\right), n=2, \ldots, N-1, \\
& \underset{(I-1) \times N+1}{\mathbf{F}_{L, 1}}=\left(\begin{array}{ccc}
F_{L, 1} & F_{L, 2} & \underset{(I-1) \times 1}{\mathbf{0}} \\
(I-1) \times 1 & (I-1) \times(N-1)
\end{array}\right), \\
& \underset{(I-1) \times N+1}{\mathbf{F}_{R, 1}}=\left(\begin{array}{ccc}
F_{R, 1} & F_{R, 2} & \underset{(I-1) \times 1}{\mathbf{0}} \\
(I-1) \times 1 & (I-1) \times(N-1)
\end{array}\right), \\
& \underset{(I-1) \times N+1}{\mathbf{F}_{L, N}}=\left(\begin{array}{ccc}
\underset{(I-1) \times(N-1)}{\mathbf{0}} & F_{L, 1} & F_{L, 2} \\
(I-1) \times 1 & (I-1) \times 1
\end{array}\right), \\
& \underset{(I-1) \times N+1}{\mathbf{F}_{R, N}}=\left(\begin{array}{ccc}
\underset{(I-1) \times(N-1)}{\mathbf{0}} & F_{R, 1} & F_{R, 2} \\
(I-1) \times 1 & (I-1) \times 1
\end{array}\right) .
\end{aligned}
$$

Then, the solution of (9) is given by:

$$
\mathbf{u}_{n+1}=B \mathbf{u}_{n}+\left(M+\Delta t S_{\boldsymbol{\theta}}\right)^{-1}\left(\mathbf{F}_{L, n} \mathbf{T}_{L}+\mathbf{F}_{R, n} \mathbf{T}_{R}\right)
$$

Applying recursively the previous relation we derive the discrete representation (Duhamel's formula)

$$
\mathbf{u}_{n}=\mathbf{B}^{n} \mathbf{u}_{0}+\sum_{k=1}^{n} \mathbf{B}^{n-k}\left(M+\Delta t S_{\boldsymbol{\theta}}\right)^{-1}\left(\mathbf{F}_{L, k} \mathbf{T}_{L}+\mathbf{F}_{R, k} \mathbf{T}_{R}\right)
$$

Now we can build the matrices $A_{n}(\boldsymbol{\theta}), \tilde{A}_{L, n}(\boldsymbol{\theta})$ and $\tilde{A}_{R, n}(\boldsymbol{\theta}), n=1, \ldots, N$, introduced in the expression (14), to recover the solution of the problem (9) as a linear function of the initial-boundary conditions, namely:

$$
\begin{aligned}
A_{n}(\boldsymbol{\theta}) & =\mathbf{B}^{n}, \\
\tilde{A}_{L, n}(\boldsymbol{\theta}) & =\sum_{k=1}^{n} \mathbf{B}^{n-k}\left(M+\Delta t S_{\boldsymbol{\theta}}\right)^{-1} \mathbf{F}_{L, k}, \text { and } \\
\tilde{A}_{R, n}(\boldsymbol{\theta}) & =\sum_{k=1}^{n} \mathbf{B}^{n-k}\left(M+\Delta t S_{\boldsymbol{\theta}}\right)^{-1} \mathbf{F}_{R, k} .
\end{aligned}
$$

\section{Appendix B}

Proof of Theorem 8:

From equations (8) and (14), we can write:

$$
\mathbf{T}_{n}=\mathbf{B}^{n} \mathbf{u}_{0}+\tilde{A}_{L, n}(\boldsymbol{\theta}) \mathbf{T}_{L}+\tilde{A}_{R, n}(\boldsymbol{\theta}) \mathbf{T}_{R}-T_{L, n} F_{L, 1}-T_{R, n} F_{R, 1},
$$


Now, define $A_{L, n}(\boldsymbol{\theta})$ and $A_{R, n}(\boldsymbol{\theta})$ by:

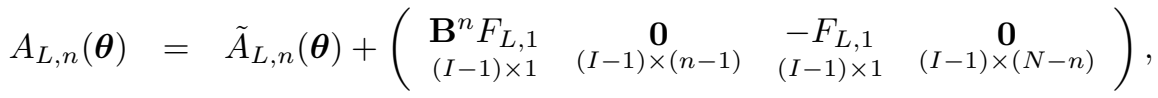

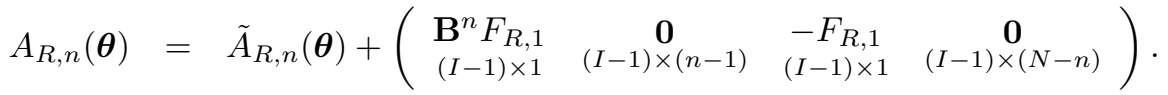

Therefore, we obtain equation (15).

\section{Appendix C}

Proof of Remark 14:

Consider the following backward Euler discretization of the local problem

(3) in the interval time, $\left(t_{n}, t_{n+1}\right)=(n \Delta t,(n+1) \Delta t)$ :

$$
\left\{\begin{aligned}
\frac{1}{\Delta t}\left(T_{i, n+1}-\right. & \left.T_{i, n}\right)-\frac{\theta}{\Delta x^{2}}\left(T_{i+1, n+1}-2 T_{i, n+1}+T_{i-1, n+1}\right)=0, \quad i=2, \ldots, I \\
T_{L, n} & =T_{L}(n \Delta t) \\
T_{R, n} & =T_{R}(n \Delta t) .
\end{aligned}\right.
$$

To write the discretization in a matrix form, let us introduce the vectors $\underset{(I-1) \times 1}{\mathbf{T}_{n}}=$ $\left(T_{2, n}, \ldots, T_{I, n}\right)^{t r}, n=1, \ldots, N$, and the matrix

$$
\underset{(I-1) \times(I-1)}{\mathbf{A}}=\left(\begin{array}{cccccc}
-2 & 1 & 0 & 0 & \ldots & 0 \\
1 & -2 & 1 & 0 & \ldots & 0 \\
0 & 1 & -2 & 1 & \ldots & 0 \\
\vdots & \ddots & \ddots & \ddots & \ddots & \vdots \\
0 & \ldots & 0 & 1 & -2 & 1 \\
0 & 0 & 0 & \ldots & 1 & -2
\end{array}\right) .
$$

In this way, we may write

$$
\frac{1}{\Delta t}\left(\mathbf{T}_{n+1}-\mathbf{T}_{n}\right)-\frac{\theta}{\Delta x^{2}} \mathbf{A} \mathbf{T}_{n+1}=\frac{\theta}{\Delta x^{2}}\left(T_{L, n+1} \mathbf{v}+T_{R, n+1} \mathbf{w}\right),
$$

where $\underset{(I-1) \times 1}{\mathbf{v}}=(1,0, \ldots, 0)^{t r}$ and $\underset{(I-1) \times 1}{\mathbf{w}}=(0, \ldots, 0,1)^{t r}$.

The expression $(\star)$ is equal to

$$
\left(I_{I-1}-\theta \frac{\Delta t}{\Delta x^{2}} \mathbf{A}\right) \mathbf{T}_{n+1}=\mathbf{T}_{n}+\theta \frac{\Delta t}{\Delta x^{2}}\left(T_{L, n+1} \mathbf{v}+T_{R, n+1} \mathbf{w}\right)
$$

and letting $\frac{\Delta t}{\Delta x^{2}}=\lambda$ and $\mathbf{B}=\left(I_{I-1}-\theta \lambda \mathbf{A}\right)^{-1}$, we obtain

$$
\mathbf{T}_{n+1}=\mathbf{B} \mathbf{T}_{n}+\theta \lambda\left(T_{L, n+1} \mathbf{B v}+T_{R, n+1} \mathbf{B w}\right) .
$$

Applying recursively the previous relation, we derive

$$
\mathbf{T}_{n}=\mathbf{B}^{n} \mathbf{T}_{0}+\theta \lambda \sum_{k=1}^{n} T_{L, k} \mathbf{B}^{n-k+1} \mathbf{v}+\theta \lambda \sum_{k=1}^{n} T_{R, k} \mathbf{B}^{n-k+1} \mathbf{w},
$$


whose compact matrix form is

$$
\mathbf{T}_{n}=\mathbf{B}^{n} \mathbf{T}_{0}+\mathbf{C}_{n} \tilde{\mathbf{T}}_{L}+\mathbf{D}_{n} \tilde{\mathbf{T}}_{R},
$$

where

- $\underset{n \times 1}{\tilde{\mathbf{T}}_{L}}=\left(T_{L, 1}, \ldots, T_{L, n}\right)^{t r}=\left(T_{L}(\Delta t), \ldots, T_{L}(n \Delta t)\right)^{t r}$,

- $\underset{n \times 1}{\tilde{\mathbf{T}}_{R}}=\left(T_{R, 1}, \ldots, T_{R, n}\right)^{t r}=\left(T_{R}(\Delta t), \ldots, T R(n \Delta t)\right)^{t r}$,

- the matrix $\underset{(I-1) \times n}{\mathbf{C}_{n}}$ has column vectors $\mathbf{c}_{k}=\theta \lambda \mathbf{A}^{n-k+1} \mathbf{v}, k=1, \ldots, n$,

- the matrix $\underset{(I-1) \times n}{\mathbf{D}_{n}}$ has column vectors $\mathbf{d}_{k}=\theta \lambda \mathbf{A}^{n-k+1} \mathbf{w}, k=1, \ldots, n$.

Now, we can build the matrices $A_{L, n}(\theta)$ and $A_{R, n}(\theta), n=1, \ldots, N$, introduced $(I-1) \times N \quad(I-1) \times N$

in the expression (16), to recover the solution of the problem (3) for each interval time, $\left(t_{n-1}, t_{n}\right), n=1, \ldots, N$, as a linear function of the initial-boundary conditions:

$$
\begin{aligned}
& \underset{(I-1) \times N}{A_{L, n}(\theta)}=\left(\begin{array}{cc}
\underset{\mathbf{C}_{n}}{\mathbf{0}} & \mathbf{0} \\
(I-1) \times n & (I-1) \times(N-n)
\end{array}\right), \\
& \underset{(I-1) \times N}{A_{R, n}(\theta)}=\left(\begin{array}{cc}
\mathbf{C}_{n} & \mathbf{0} \\
(I-1) \times n & (I-1) \times(N-n)
\end{array}\right) .
\end{aligned}
$$

